\title{
Oral premedication in patients with a history suggesting hypersensitivity to iodinated contrast media
}

\author{
Krzysztof Specjalski, Lucyna Górska, Beata Wajda, Marta Chełmińska, Ewa Jassem
}

Department of Allergology, Medical University of Gdansk, Gdansk, Poland

Adv Dermatol Allergol 2020; XXXVII (4): 520-523

DOI: https://doi.org/10.5114/ada.2020.98269

\begin{abstract}
Introduction: lodinated contrast media (ICM) are pharmaceuticals widely used in diagnostic procedures. Adverse effects associated with their administration are quite frequent and mostly mild. However, they raise concerns in patients and doctors in the context of their future use.

Aim: To determine efficacy of premedication before medical procedures with the use of iodinated contrast media in patients with a history suggesting a hypersensitivity reaction after their past use.

Material and methods: Out of 152 patients consulted due to adverse reactions after ICM (85 women and 67 men, aged 43-90), 101 were selected with the history suggesting a mild hypersensitivity reaction (urticaria, itching, skin redness, malaise etc.). All the patients had health problems requiring a procedure with ICM administration in the near future. The premedication was given with cetirizine $(10 \mathrm{mg})$ and prednisone $(20 \mathrm{mg}$ or $50 \mathrm{mg}$, randomly assigned) 13,7 and $1 \mathrm{~h}$ before the ICM administration. Presence of adverse events was compared between the subgroups with $\chi^{2}$ test and efficacy of premedication - with Wilcoxon test.

Results: Seventy-six patients underwent the radiologic procedure with premedication with antihistamine and a lower (40 patients) or higher dose (36 patients) of prednisone. Four of them reported a cutaneous hypersensitivity reaction (urticaria, itching, redness) and one - dyspnoea. There was no statistically significant difference in relation to the premedication protocol $(p=0.1306)$.

Conclusions: Premedication with cetirizine and prednisone before radiologic procedures proved to be efficient in patients with a history suggesting hypersensitivity to iodinated contrast media. There was no significant difference in efficacy related to the dose of prednisone $(20 \mathrm{mg}$ vs. $50 \mathrm{mg}$ ).
\end{abstract}

Key words: iodinated contrast media, contrast media allergy, drug hypersensitivity, premedication.

\section{Introduction}

lodinated contrast media (ICM) are solutions of iodinated benzene derivatives widely used in diagnostic procedures. It is estimated they are administered more than 75 million times every year in several X-ray procedures [1]. In Poland itself the number of coronary angiographies exceeds 200000 per year including more than 120000 percutaneous coronary interventions [2].

Adverse effects associated with ICM administration are quite frequent and mostly non-allergic. The risk of adverse reactions is $4-12 \%$ with ionic ICM and $1-3 \%$ with nonionic contrast media. Severe reactions are observed in $0.16 \%$ and $0.03 \%$ of procedures, respectively [3, 4]. However, the reactions, particularly milder and not requiring interventions, may be significantly underestimated.
Although majority of the reactions are mild, they raise concerns in patients and doctors in the context of their future use. Thus, an uncertain history of 'allergy to contrast media' may lead to unnecessary avoidance of important or even life-saving procedures, for example coronary angiography and percutaneous coronary intervention ( $\mathrm{PCl}$ ). As a consequence, it seems necessary to introduce reliable and evidence-based protocols of management of patients with several patterns of adverse reactions to ICM in history.

\section{Aim}

The aim of the study was to determine efficacy of premedication before medical procedures with the use of io-

Address for correspondence: Krzysztof Specjalski MD, PhD, Department of Allergology, Medical University of Gdansk, 7 Dębinki St, 80-952 Gdańsk, Poland, phone: +48 608631 547, e-mail: specjalski@gumed.edu.pl Received: 1.08.2018, accepted: 1.01.2019. 
dinated contrast media in patients with a history suggesting a mild hypersensitivity reaction after their past use.

\section{Material and methods}

Between January 2015 and January 2018, 152 patients (85 women and 67 men, aged 40-90 years) were consulted in the Department of Allergology, Medical University of Gdansk due to the past hypersensitivity reactions after ICM. Out of 152 patients, a group of 101 was selected with the history suggesting a mild hypersensitivity reaction (urticaria, itching, angioedema etc.). All the participants had health problems requiring a procedure with ICM administration in the near future (coronary angiography or computed tomography). Patients with the history of a severe drug hypersensitivity reaction, including anaphylaxis as defined by Sampson [5], unstable asthma, renal insufficiency or unstable heart insufficiency were excluded from the study. We also excluded patients with isolated subjective vasomotor symptoms (nausea, sweating, feeling of warmth etc.).

Patients were randomly assigned to one of the premedication arms: $10 \mathrm{mg}$ cetirizine $+20 \mathrm{mg}$ prednisone or $10 \mathrm{mg}$ cetirizine $+50 \mathrm{mg}$ prednisone. Characteristics of the subgroups are presented in Table 1 . The premedica- tion was given orally 13,7 and $1 \mathrm{~h}$ before the ICM administration. Subjects were observed $24 \mathrm{~h}$ after the ICM administration. Presence of any hypersensitivity reactions was recorded.

The protocol of the study has been approved by the Independent Bioethics Committee of the Medical University of Gdansk. Subjects have given written informed consent before inclusion into the study.

\section{Statistical analysis}

In statistical analysis their number was compared between the groups with $\chi^{2}$ test. Efficacy of premedication expressed by reduction of adverse events was determined with Wilcoxon test (Statistica 13, StatSoft, USA).

\section{Results}

Out of 101 patients recruited into the study, 76 patients underwent radiologic procedures with premedication with antihistamine and a lower (40 patients) or higher dose (36 patients) of prednisone. Coronary angiography was performed in 67 and computed tomography - in 9 cases.

In the vast majority of cases (70 out of 76 , i.e. 92\%) diagnostic procedures were performed without any com-

Table 1. Characteristics of subgroups based on the premedication protocol applied: A - cetirizine + prednisone (low dose), $\mathrm{B}$ - cetirizine + prednisone (high dose)

\begin{tabular}{|c|c|c|}
\hline Parameter & Subgroup A $(n=40)$ & Subgroup B $(n=36)$ \\
\hline Age (mean) & $53-82$ years ( 48.9 years) & $40-90$ years ( 46.5 years) \\
\hline Gender (women : men) & $19: 21$ & $21: 15$ \\
\hline \multicolumn{3}{|c|}{ Concomitant allergic diseases, $n(\%)$ : } \\
\hline Asthma & $1(2.5)$ & $2(5.5)$ \\
\hline Allergic rhinitis & $5(12.5)$ & $3(8.3)$ \\
\hline Chronic urticaria & $0(0)$ & $1(2.7)$ \\
\hline Atopic dermatitis & $1(2.5)$ & $0(0)$ \\
\hline \multicolumn{3}{|c|}{ History of reactions to ICMs, $n$ (\%): } \\
\hline Urticaria & $26(65)$ & $19(52)$ \\
\hline Angioedema & $8(20)$ & $9(25)$ \\
\hline Skin redness & $29(72.5)$ & $26(72)$ \\
\hline Nausea & $6(15)$ & $4(11)$ \\
\hline Diarrhoea & $4(10)$ & $3(8.3)$ \\
\hline Dizziness & $3(7.5)$ & $4(11)$ \\
\hline \multicolumn{3}{|c|}{ Procedure associated with the past reaction to ICMs, $n(\%)$ : } \\
\hline Coronary angiography & $20(50)$ & $16(44)$ \\
\hline Computed tomography & $6(15)$ & $6(16)$ \\
\hline Urography & $8(20)$ & $6(16)$ \\
\hline Other & $2(5)$ & $8(22)$ \\
\hline Lack of data & $4(11)$ & $3(8.3)^{*}$ \\
\hline
\end{tabular}

*Sum higher than 36 - some patients had more than one reaction. 


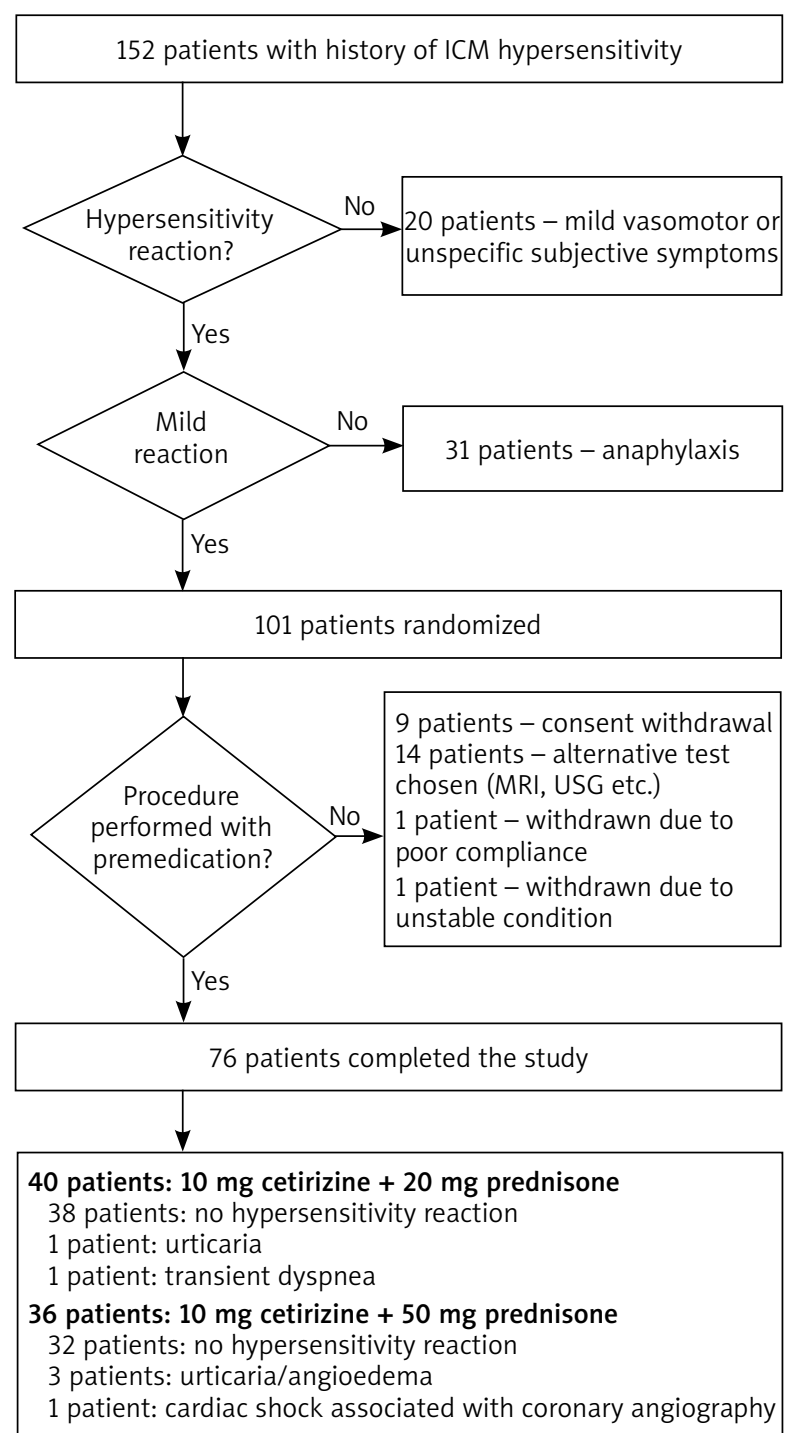

Figure 1. Patients flow from the recruitment to assessment of premedication efficacy

plications. In 4 (5\%) patients a cutaneous hypersensitivity reaction was observed (urticaria, itching, redness). One patient had a transient dyspnoea during the procedure with no other symptoms suggesting hypersensitivity. Finally, 1 patient had cardiac arrest during angiography, with no typical signs and symptoms of hypersensitivity, probably associated with the procedure itself. Thus, this case was excluded from the analysis. The reduction in the number of hypersensitivity symptoms between the patient's history and current ICM administration was statistically significant $(p<0.01)$ while the difference in the rate of reactions between premedication groups was non-significant $(p=0.1306)$. The flow of patients has been shown in Figure 1.

\section{Discussion}

Similarly to drugs hypersensitivity, reactions to ICM are generally classified as immediate (onset of the reaction within $1 \mathrm{~h}$ after administration) and delayed (up to 24-48 $\mathrm{h}$ after administration). Immediate reactions are usually more severe with generalized symptoms (most commonly - urticaria, angioedema, dyspnoea, wheezing, fall of blood pressure) while delayed reactions are milder, usually limited to the skin [6]. For many years immediate reactions were linked to nonspecific histamine release resulting from a direct membrane effect of highosmolality ICM, activation of complement or formation of bradykinin. However, there is a growing evidence that some of the reactions are IgE-mediated allergy [7]. Nonimmediate allergic reactions are T-cell-dependent.

There are no widely accepted standards for the future use of ICM in patients with the history of adverse events. If the hypersensitivity reaction had been severe, administration of ICM is for obvious reasons strictly forbidden. On the other hand, it is believed that patients presenting mild vasomotor symptoms do not need any particular attention as they mostly well tolerate new lower-osmolality ICM. Management of mild-to-moderate hypersensitive patients has been more controversial. For many years their reactions were regarded as 'anaphylactoid' i.e. non-IgE-mediated. Thus, skin tests were not advised as unreliable. This approach has been changing as the increasing number of publications show that in patients demonstrating typical signs and symptoms of immediate, anaphylactic reactions, skin tests reflect sensitization [8]. As a result, the skin test, together with challenges may be applied for finding a safe ICM for future use [9]. In the vast majority of cases both immediate and non-immediate ICM-related reactions are mild. If patients' condition requires a diagnostic or therapeutic procedure with the use of ICM and the benefit/risk ratio is clear, pretreatment is also often used. According to the current guidelines of the Polish Society of Allergology, pretreatment is particularly efficient in patients with the history of a mild reaction [10].

In order to minimize the incidence of hypersensitivity reactions in high-risk patients, several premedication protocols have been proposed, mostly based on administration of glucocorticosteroids and antihistamines. In the study of Greenberger et al., 563 patients with the history of immediate hypersensitivity reactions were given either the combination of $50 \mathrm{mg}$ prednisone, $50 \mathrm{mg}$ diphenhydramine and $25 \mathrm{mg}$ ephedrine or $50 \mathrm{mg}$ prednisone and $50 \mathrm{mg}$ diphenhydramine. Both regimens were assessed to be efficient with a significantly lower number of mild reactions in the group receiving ephedrine [11]. Two years later Greenberger et al. proposed another regime for emergency administration of ICM with $200 \mathrm{mg}$ hydrocortisone given intravenously repeatedly every $4 \mathrm{~h}$ 
until the end of the procedure and $50 \mathrm{mg}$ diphenhydramine intravenously $1 \mathrm{~h}$ before the procedure [12]. Later introduction of lower-osmolality ICM proved to be safer in high-risk patients [13].

Although well documented, the presented regimens cannot be easily implemented. Diphenhydramine is in many counties no longer available in the oral form and the second generation of antihistamines prevail on the market due to a lower incidence of unwanted effects. Ephedrine is contraindicated in patients with hypertension and coronary artery disease. Another issue may be a relatively high dose of prednisone $(150 \mathrm{mg} /$ day) proposed. Although the risk of adverse events in short-term administration of systemic steroids is not high, some patients with chronic diseases, such as diabetes or hypertension, may suffer from the deterioration of their control.

Our regimens are based on oral, easily-available drugs that can be taken by patients at home and, as a result, earlier hospitalization is not required for pretreatment. This approach proved to be efficient with only 5 patients demonstrating relatively mild hypersensitivity symptoms.

The main limitation of the study is selection of patients only with the history of mild hypersensitivity reactions. Thus, results cannot be simply extrapolated to the whole population of ICM-intolerant patients. On the other hand, regarding several patterns of reactivity and numerous clinical situations, the diagnostic approach cannot be unified. On the contrary, it should be patienttailored with either pretreatment or allergology work-up aiming at finding safe alternative ICM [6]. For ethical reasons, no placebo group was designed, what affected the reliability of efficacy assessment. Presence of hypersensitivity reactions was compared between patients' history and current study despite the fact that different ICMS could have been applied.

Another not investigated area is the relation between adverse events and general condition of patients as well as concomitant drugs. In this study all the procedures were elective and, as a result, performed in stable and prepared patients. That does not reflect real life where ICM are often administered in emergency situations and hypersensitivity reactions may be augmented by cardiac insufficiency, $\beta$-blockers administration etc.

\section{Conclusions}

Premedication with cetirizine and prednisone before radiologic procedures proved to be efficient in patients with a history suggesting hypersensitivity to iodinated contrast media irrespective of the dose of prednisone (20 mg vs. $50 \mathrm{mg}$ ).

\section{Acknowledgments}

Publication of the article financed by ST-554 Gdansk Medical University.

\section{Conflict of interest}

The authors declare no conflict of interest.

\section{References}

1. Christiansen C. X-ray contrast media - an overview. Toxicology 2005; 209: 185-7.

2. Dudek D, Legutko J, Siudak Z, et al. Kardiologia interwencyjna w Polsce w 2012 roku. Raport Zarządu Interwencji Sercowo-Naczyniowych Polskiego Towarzystwa Kardiologicznego. Kardiol Pol 2013; 71: 1213-9.

3. Kaufman D. Risk of anaphylaxis in a hospital population in relation to the use of various drugs: an international study. Pharmacoepidemiol Drug Saf 2003; 12: 195-202.

4. Cochran S. Anaphylactoid reactions to radiocontrast media. Curr Allergy Asthma Rep 2005; 5: 28-31.

5. Sampson H, Munoz-Furlong A, Campbell R, et al. Second symposium on the definition and management of anaphylaxis: summary report. J Allergy Clin Immunol 2006; 117: 391-7.

6. Rosado A, Dona I, Cabanas M, et al. Clinical practice guidelines for diagnosis and management of hypersensitivity reactions to contrast media. J Invest Allergol Clin Immunol 2016; 26: 144-55.

7. Brockow K, Romano A, Aberer W, et al. Skin testing in patients with hypersensitivity reactions to iodinated contrast media - a European multicenter study. Allergy 2009; 64: 234-41.

8. Yoon S, Lee S, Kang H, et al. Skin test in patients with hypersensitivity reaction to iodinated contrast media: a metaanalysis. Allergy 2015; 70: 625-37.

9. Sese L, Gaouar H, Autegarde J, et al. Immediate hypersensitivity to iodinated contrast media: diagnostic accuracy of skin tests and intravenous provocation test with low dose. Clin Exp Allergy 2016; 46: 472-8.

10. Nitter-Marszalska M, Bochenek G, Bodzenta-tukaszyk A, et al. Hypersensitivity to iodine contrast media. Guidelines of the Section of Drug Hypersensitivity of the Polish Society of Allergology. Pol J Allergol 2018; 5: 51-7.

11. Greenberger P, Patterson R, Radin R. Two pretreatment regimens for high-risk patients receiving radiographic contrast media. J Allergy Clin Immunol 1984; 74: 540-3.

12. Greenberger P, Halwig J, Patterson R, et al. Emergency administration of radiocontrast media in high-risk patients. J Allergy Clin Immunol 1986; 77: 630-4.

13. Greenberger P, Patterson R. The prevention of immediate generalized reactions to radiocontrast media in high-risk patients. J Allergy Clin Immunol 1991; 87: 867-72. 